\title{
Analytical Results for MOX Colemanite Concrete Samples Received on January 15, 2013
}

M.M. Reigel

D.R. Best

February 2013

Savannah River National Laboratory Savannah River Nuclear Solutions, LLC Aiken, SC 29808

Prepared for the U.S. Department of Energy under contract number DE-AC09-08SR22470. 
SRNL-STI-2013-00078

Revision 0

\section{DISCLAIMER}

This work was prepared under an agreement with and funded by the U.S. Government. Neither the U.S. Government or its employees, nor any of its contractors, subcontractors or their employees, makes any express or implied:

1. warranty or assumes any legal liability for the accuracy, completeness, or for the use or results of such use of any information, product, or process disclosed; or

2. representation that such use or results of such use would not infringe privately owned rights; or

3. endorsement or recommendation of any specifically identified commercial product, process, or service.

Any views and opinions of authors expressed in this work do not necessarily state or reflect those of the United States Government, or its contractors, or subcontractors.

\section{Printed in the United States of America}

Prepared for

U.S. Department of Energy 
Keywords: $M O X$

Colemanite Concrete

Retention: Permanent

\section{Analytical Results for MOX Colemanite Concrete Samples Received on January 15, 2013}

M.M. Reigel
D.R. Best

February 2013

Savannah River National Laboratory Savannah River Nuclear Solutions, LLC Aiken, SC 29808

Prepared for the U.S. Department of Energy under contract number DE-AC09-08SR22470. 


\section{REVIEWS AND APPROVALS}

AUTHORS:

M.M. Reigel, Engineering Process Development

Date

D.R. Best, Engineering Process Development

Date

TECHNICAL REVIEW:

A.D. Cozzi, Engineering Process Development

Date

APPROVAL:

E.N. Hoffman, Manager

Date

Engineering Process Development

S.L. Marra, Manager

Date

Environmental \& Chemical Process Technology Research Programs 


\section{EXECUTIVE SUMMARY}

The Mixed Oxide Fuel Fabrication Facility (MFFF) will use colemanite bearing concrete neutron absorber panels credited with attenuating neutron flux in the criticality design analyses and shielding operators from radiation. The Savannah River National Laboratory (SRNL) is tasked with measuring the total density, partial hydrogen density, and partial boron density of the colemanite concrete.

SRNL received twelve samples of colemanite concrete for analysis on January 15, 2013. The average total density of each of the samples measured by the ASTM method C 642, the average partial hydrogen density was measured using method ASTM E 1311, and the average partial boron density of each sample was measured according to ASTM C 1301. The lower limits and measured values for the total density, hydrogen partial density, and boron partial density are presented in the following table. For all the samples tested, the total density and the hydrogen partial density met or exceeded the specified limit. All of the samples met or exceeded the boron partial density lower bound with the exception of samples G3-M11-2000-H, G3-M11-3000-M, and G5-M1-3000-H which are below the limit of 1.65E-01 g/ $\mathrm{cm}^{3}$.

\begin{tabular}{|c|c|c|c|c|c|c|}
\hline \multirow[t]{2}{*}{ Sample ID } & \multicolumn{2}{|c|}{$\begin{array}{c}\text { Total Density Lower } \\
\text { Bound } \\
{\left[\mathrm{g} / \mathrm{cm}^{3}\right]}\end{array}$} & \multicolumn{2}{|c|}{$\begin{array}{c}\text { Hydrogen Partial } \\
\text { Density Lower Bound } \\
{\left[\mathrm{g} / \mathrm{cm}^{3}\right]}\end{array}$} & \multicolumn{2}{|c|}{$\begin{array}{c}\text { Boron Partial Density } \\
\text { Lower Bound } \\
{\left[\mathrm{g} / \mathrm{cm}^{3}\right]}\end{array}$} \\
\hline & Limit & Measured & Limit & Measured & Limit & Measured \\
\hline G3-M11-2000-B & \multirow{12}{*}{1.88} & 2.03 & \multirow{12}{*}{$6.04 \mathrm{E}-02$} & 7.12E-02 & \multirow{12}{*}{$1.65 \mathrm{E}-01$} & 1.70E-01 \\
\hline G3-M11-2000-M & & 2.04 & & 7.25E-02 & & $1.79 \mathrm{E}-01$ \\
\hline G3-M11-2000-H & & 2.02 & & 7.73E-02 & & $1.61 \mathrm{E}-01$ \\
\hline G3-M11-3000-B & & 2.00 & & 6.65E-02 & & $1.69 \mathrm{E}-01$ \\
\hline G3-M11-3000-M & & 2.02 & & 7.33E-02 & & $1.64 \mathrm{E}-01$ \\
\hline G3-M11-3000-H & & 2.06 & & $7.26 \mathrm{E}-02$ & & $1.70 \mathrm{E}-01$ \\
\hline G4-M1-2000-B & & 2.03 & & $6.81 \mathrm{E}-02$ & & 1.72E-01 \\
\hline G4-M1-2000-M & & 2.03 & & 6.85E-02 & & 1.67E-01 \\
\hline G4-M1-2000-H & & 2.05 & & $6.88 \mathrm{E}-02$ & & $1.72 \mathrm{E}-01$ \\
\hline G5-M1-3000-B & & 2.03 & & 7.47E-02 & & $1.74 \mathrm{E}-01$ \\
\hline G5-M1-3000-M & & 2.03 & & 7.42E-02 & & $1.66 \mathrm{E}-01$ \\
\hline G5-M1-3000-H & & 2.03 & & $7.24 \mathrm{E}-02$ & & $1.59 \mathrm{E}-01$ \\
\hline
\end{tabular}




\section{TABLE OF CONTENTS}

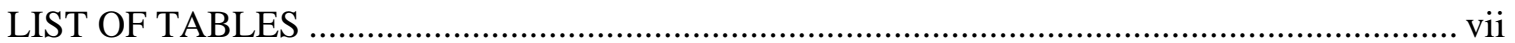

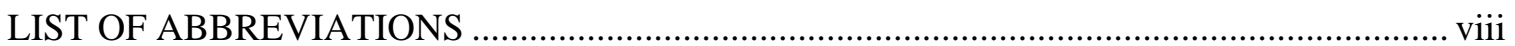

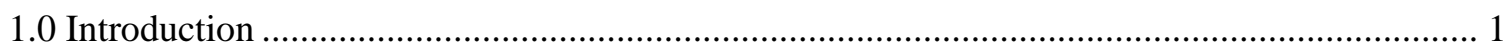

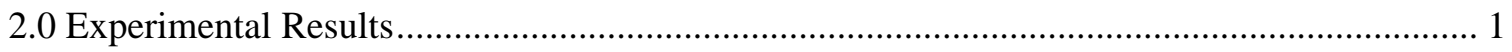

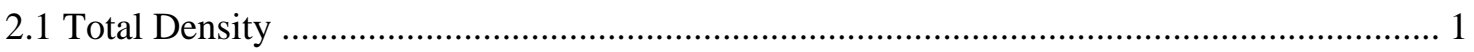

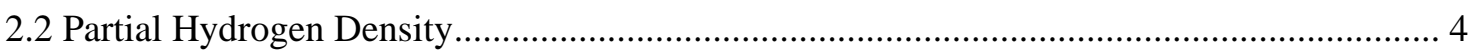

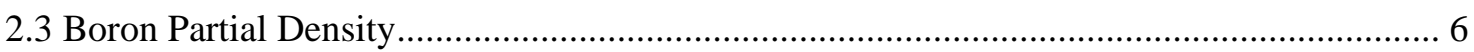

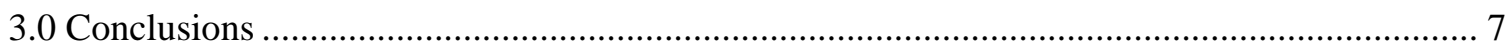

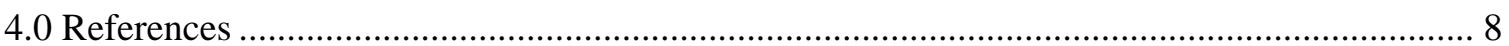




\section{LIST OF TABLES}

Table 1-1. Acceptable Material Neutron Absorber Characteristics................................................ 1

Table 2-1. Mass measurements of duplicate samples after each treatment ................................... 2

Table 2-2. Calculated results using equations $1-7$ for colemanite concrete samples received

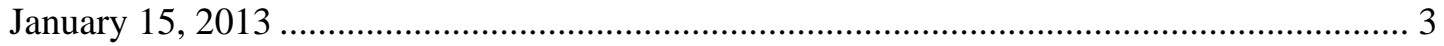

Table 2-3. Mass loss from $25-650^{\circ} \mathrm{C}$ for samples received on January 15, 2013........................ 5

Table 2-4. Boron results for colemanite concrete samples received January 15, 2013 .................. 6

Table 3-1. Lower Bounds and Average Measured Values for the Total Density, Hydrogen Partial Density, and Boron Partial Density for the Colemanite Concrete Samples Received January

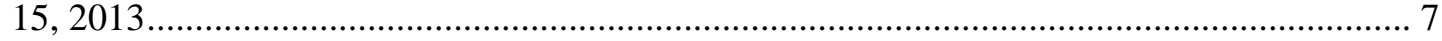




\section{LIST OF ABBREVIATIONS}

$\begin{array}{ll}\text { ASTM } & \text { American Society for Testing and Materials } \\ \text { DSC } & \text { Differential Scanning Calorimetry } \\ \text { ICP } & \text { Inductively Coupled Plasma } \\ \text { MFFF } & \text { Mixed Oxide Fuel Fabrication Facility } \\ \text { OES } & \text { Optical Emission Spectrometer } \\ \text { PSAL } & \text { Process Science Analytical Laboratory } \\ \text { SRNL } & \text { Savannah River National Laboratory } \\ \text { TGA } & \text { Thermal Gravimetric Analysis }\end{array}$




\subsection{Introduction}

The Mixed Oxide Fuel Fabrication Facility (MFFF) will use colemanite bearing concrete neutron absorber panels credited with attenuating neutron flux in the criticality design analyses and shielding the operator from radiation. ${ }^{1}$ The properties listed in Table 1-1 are from Table 2.1.2.3 in Reference 1. Savannah River National Laboratory (SRNL) is tasked with measuring the properties of colemanite concrete identified in Table 1-1. ${ }^{2}$

Table 1-1. Acceptable Material Neutron Absorber Characteristics

\begin{tabular}{|c|c|c|c|}
\hline Material Type & $\begin{array}{c}\text { Total Density Lower } \\
\text { Bound }\left(\mathrm{g} / \mathrm{cm}^{3}\right)\end{array}$ & $\begin{array}{c}\text { Hydrogen Partial Density } \\
\text { Lower Bound }\left(\mathrm{g} / \mathrm{cm}^{3}\right) \\
\end{array}$ & $\begin{array}{l}\text { Boron Partial Density } \\
\text { Lower Bound }\left(\mathrm{g} / \mathrm{cm}^{3}\right) \\
\end{array}$ \\
\hline $\begin{array}{l}\text { Borated Concrete } \\
\text { (Colemanite) }\end{array}$ & 1.88 & 6.04E-02 & $1.65 \mathrm{E}-01$ \\
\hline
\end{tabular}

\subsection{Experimental Results}

Twelve samples of colemanite concrete were delivered to SRNL on January 15, 2013. All samples were cuboids with approximate dimensions of $160 \mathrm{~mm}$ x $40 \mathrm{~mm}$ x $40 \mathrm{~mm}$. The samples arrived in zip top bags and each sample was wrapped in a moist towel.

\subsection{Total Density}

The total density of each sample was determined according to ASTM standard C 642-06 for determining the density of hardened concrete. ${ }^{3}$ The ASTM method was followed with the exception of sample size. Section 4.1 of the ASTM procedure specifies a sample size of approximately 800 grams. As the samples themselves weighed less than 550 grams, a reduced sample size was used. Duplicate samples for total density were obtained by cleaving pieces from the as-received sample. Mass measurements were taken after each treatment (Table 2-1) as outlined in the ASTM standard with the masses designated as A through D, where:

$$
\begin{aligned}
& A=\text { mass of oven-dried sample in air between } 100 \text { to } 110{ }^{\circ} \mathrm{C}, \mathrm{g} \\
& \mathrm{B}=\text { mass of surface-dry sample in air after immersion, } \mathrm{g} \\
& \mathrm{C}=\text { mass of surface-dry sample in air after immersion and boiling, } \mathrm{g} \\
& \mathrm{D}=\text { apparent mass of sample in water after immersion and boiling, } \mathrm{g}
\end{aligned}
$$

Using the calculations in the ASTM method, the following properties were calculated (Table 2-2):

Absorption after immersion, $\%=\frac{B-A}{A} \times 100$,

Absorption after immersion and boiling, $\%=\frac{C-A}{A} \times 100$,

Bulk density, dry $=\frac{A}{C-D} \times \rho$,

Bulk density after immersion $=\frac{B}{C-D} \times \rho$,

Bulk density after immersion and boiling $=\frac{C}{C-D} \times \rho$,

Apparent density $=\frac{A}{A-D} \times \rho$,

Volume of permeable pore space $=\frac{C-A}{C-D} \times 100$ 
SRNL-STI-2013-00078

Revision 0

Table 2-1. Mass measurements of duplicate samples after each treatment

\begin{tabular}{|c|c|c|c|c|c|}
\hline Sample ID & $\begin{array}{c}\text { Run } \\
\text { Number }\end{array}$ & $\begin{array}{c}\text { Dry (A) } \\
\text { [g] }\end{array}$ & $\begin{array}{c}\text { Saturated (B) } \\
\text { [g] }\end{array}$ & $\begin{array}{c}\text { Boiled (C) } \\
\text { [g] }\end{array}$ & $\begin{array}{c}\text { Suspended (D) } \\
\text { [g] }\end{array}$ \\
\hline \multicolumn{2}{|c|}{ Date } & $1 / 22 / 2013$ & $1 / 24 / 2012$ & $1 / 29 / 2013$ & $1 / 29 / 2013$ \\
\hline \multirow{2}{*}{ G3-M11-2000-B } & 1 & 4.894 & 5.819 & 5.767 & 2.892 \\
\hline & 2 & 11.754 & 14.041 & 13.911 & 6.991 \\
\hline \multirow{2}{*}{ G3-M11-2000-M } & 1 & 11.338 & 13.439 & 13.456 & 6.842 \\
\hline & 2 & 10.592 & 12.411 & 12.228 & 6.196 \\
\hline \multirow{2}{*}{ G3-M11-2000-H } & 1 & 12.292 & 14.413 & 14.438 & 7.356 \\
\hline & 2 & 9.482 & 11.454 & 11.320 & 5.634 \\
\hline \multirow{2}{*}{ G3-M11-3000-B } & 1 & 9.549 & 11.653 & 11.627 & 5.759 \\
\hline & 2 & 16.267 & 19.685 & 19.620 & 9.842 \\
\hline \multirow{2}{*}{ G3-M11-3000-M } & 1 & 6.203 & 7.529 & 7.435 & 3.678 \\
\hline & 2 & 5.690 & 6.738 & 6.614 & 3.318 \\
\hline \multirow{2}{*}{ G3-M11-3000-H } & 1 & 13.829 & 16.604 & 16.529 & 8.367 \\
\hline & 2 & 10.857 & 12.733 & 12.640 & 6.523 \\
\hline \multirow{2}{*}{ G4-M1-2000-B } & 1 & 6.626 & 8.008 & 7.948 & 3.962 \\
\hline & 2 & 11.303 & 13.318 & 13.360 & 6.856 \\
\hline \multirow{2}{*}{ G4-M1-2000-M } & 1 & 7.953 & 8.734 & 8.770 & 4.449 \\
\hline & 2 & 10.181 & 12.088 & 12.102 & 6.170 \\
\hline \multirow{2}{*}{ G4-M1-2000-H } & 1 & 9.856 & 11.503 & 11.500 & 5.924 \\
\hline & 2 & 7.538 & 8.994 & 8.927 & 4.496 \\
\hline \multirow{2}{*}{ G5-M1-3000-B } & 1 & 8.570 & 10.315 & 10.238 & 5.169 \\
\hline & 2 & 14.021 & 16.766 & 16.740 & 8.457 \\
\hline \multirow{2}{*}{ G5-M1-3000-M } & 1 & 15.022 & 17.814 & 17.888 & 9.161 \\
\hline & 2 & 8.560 & 10.337 & 10.352 & 5.221 \\
\hline \multirow{2}{*}{ G5-M1-3000-H } & 1 & 13.480 & 15.856 & 15.772 & 8.057 \\
\hline & 2 & 10.980 & 13.356 & 13.396 & 6.705 \\
\hline
\end{tabular}

The density, $\rho$, used in these calculations is that of water, $\left(1 \mathrm{~g} / \mathrm{cm}^{3}\right)$. The results of the calculations performed with Equations 1-7 are tabulated and averaged in Table 2-2. 
Table 2-2. Calculated results using equations 1 - 7 for colemanite concrete samples received January 15, 2013

\begin{tabular}{|c|c|c|c|c|c|c|c|c|c|}
\hline Sample ID & $\begin{array}{c}\text { Run } \\
\text { Number } \\
\end{array}$ & $\begin{array}{c}\text { Eq. } 1 \\
{[\%]} \\
\end{array}$ & $\begin{array}{c}\text { Eq. } 2 \\
{[\%]} \\
\end{array}$ & $\begin{array}{c}\text { Eq. } 3 \\
{\left[\mathrm{~g} / \mathrm{cm}^{3}\right]} \\
\end{array}$ & $\begin{array}{r}\mathrm{Eq.} 4 \\
{\left[\mathrm{~g} / \mathbf{c m}^{3}\right]} \\
\end{array}$ & $\begin{array}{r}\mathbf{E q . ~} 5 \\
{\left[\mathrm{~g} / \mathrm{cm}^{3}\right]} \\
\end{array}$ & $\begin{array}{c}\mathbf{E q . ~} 6 \\
{\left[\mathrm{~g} / \mathrm{cm}^{3}\right]} \\
\end{array}$ & $\begin{array}{c}\text { Eq. } 7 \\
{[\%]} \\
\end{array}$ & $\begin{array}{c}\text { Average (Eq. 4) } \\
{\left[\mathrm{g} / \mathrm{cm}^{3}\right]}\end{array}$ \\
\hline \multirow{2}{*}{ G3-M11-2000-B } & 1 & 18.901 & 17.840 & 1.702 & 2.024 & 2.006 & 2.444 & 30.362 & \multirow{2}{*}{2.03} \\
\hline & 2 & 19.457 & 18.349 & 1.699 & 2.029 & 2.010 & 2.468 & 31.166 & \\
\hline \multirow{2}{*}{ G3-M11-2000-M } & 1 & 18.531 & 18.681 & 1.714 & 2.032 & 2.035 & 2.522 & 32.026 & \multirow{2}{*}{2.04} \\
\hline & 2 & 17.173 & 15.446 & 1.756 & 2.057 & 2.027 & 2.409 & 27.120 & \\
\hline \multirow{2}{*}{ G3-M11-2000-H } & 1 & 17.255 & 17.460 & 1.736 & 2.035 & 2.039 & 2.490 & 30.306 & \multirow{2}{*}{2.02} \\
\hline & 2 & 20.797 & 19.382 & 1.668 & 2.015 & 1.991 & 2.464 & 32.323 & \\
\hline \multirow{2}{*}{ G3-M11-3000-B } & 1 & 22.034 & 21.756 & 1.627 & 1.986 & 1.981 & 2.519 & 35.405 & \multirow{2}{*}{2.00} \\
\hline & 2 & 21.012 & 20.609 & 1.664 & 2.013 & 2.007 & 2.532 & 34.289 & \\
\hline \multirow{2}{*}{ G3-M11-3000-M } & 1 & 21.377 & 19.860 & 1.651 & 2.004 & 1.979 & 2.456 & 32.788 & \multirow{2}{*}{2.02} \\
\hline & 2 & 18.418 & 16.243 & 1.726 & 2.044 & 2.006 & 2.398 & 28.035 & \\
\hline \multirow{2}{*}{ G3-M11-3000-H } & 1 & 20.067 & 19.526 & 1.694 & 2.034 & 2.025 & 2.532 & 33.081 & \multirow{2}{*}{2.06} \\
\hline & 2 & 17.279 & 16.422 & 1.775 & 2.081 & 2.066 & 2.505 & 29.145 & \\
\hline \multirow{2}{*}{ G4-M1-2000-B } & 1 & 20.857 & 19.946 & 1.663 & 2.009 & 1.994 & 2.488 & 33.163 & \multirow{2}{*}{2.03} \\
\hline & 2 & 17.827 & 18.194 & 1.738 & 2.048 & 2.054 & 2.542 & 31.622 & \\
\hline \multirow{2}{*}{ G4-M1-2000-M } & 1 & 9.820 & 10.273 & 1.840 & 2.021 & 2.030 & 2.270 & 18.907 & \multirow{2}{*}{2.03} \\
\hline & 2 & 18.731 & 18.871 & 1.716 & 2.037 & 2.040 & 2.538 & 32.384 & \\
\hline \multirow{2}{*}{ G4-M1-2000-H } & 1 & 16.711 & 16.679 & 1.768 & 2.063 & 2.062 & 2.507 & 29.483 & \multirow{2}{*}{2.05} \\
\hline & 2 & 19.315 & 18.428 & 1.701 & 2.030 & 2.014 & 2.478 & 31.346 & \\
\hline \multirow{2}{*}{ G5-M1-3000-B } & 1 & 20.362 & 19.464 & 1.691 & 2.035 & 2.020 & 2.520 & 32.907 & \multirow{2}{*}{2.03} \\
\hline & 2 & 19.578 & 19.392 & 1.693 & 2.024 & 2.021 & 2.520 & 32.827 & \\
\hline \multirow{2}{*}{ G5-M1-3000-M } & 1 & 18.586 & 19.079 & 1.721 & 2.041 & 2.050 & 2.563 & 32.840 & \multirow{2}{*}{2.03} \\
\hline & 2 & 20.759 & 20.930 & 1.668 & 2.015 & 2.018 & 2.564 & 34.920 & \\
\hline \multirow{2}{*}{ G5-M1-3000-H } & 1 & 17.626 & 17.001 & 1.747 & 2.055 & 2.044 & 2.486 & 29.707 & \multirow{2}{*}{2.03} \\
\hline & 2 & 21.639 & 21.999 & 1.641 & 1.996 & 2.002 & 2.568 & 36.103 & \\
\hline
\end{tabular}




\subsection{Partial Hydrogen Density}

The hydrogen partial density of the colemanite concrete was determined using the ASTM method for determining volatile content using thermogravimetric analysis. ${ }^{4}$ The ASTM E 1131-08 defines highly volatile matter as components that will volatilize $<200^{\circ} \mathrm{C}$ and medium volatile matter as components that will degrade or volatilize in the range $200-750{ }^{\circ} \mathrm{C}$. In an evaluation of the thermal decomposition of colemanite, Waclawska et al, determined that the release of water from colemanite was complete at $600{ }^{\circ} \mathrm{C}$ and that melting and crystallization of a calcium borate phase occurred above $650{ }^{\circ} \mathrm{C} . .^{5}$ A Netzsch STA 409 Luxx, which couples Differential Scanning Calorimetry (DSC) with Thermal Gravimetric Analysis (TGA), was used for determining the partial hydrogen density of the colemanite concrete samples. After loading the sample, the chamber was purged with nitrogen at $60 \mathrm{ml} / \mathrm{min}$ prior to heating. Triplicate samples were heated at $5{ }^{\circ} \mathrm{C} / \mathrm{min}$ up to $650{ }^{\circ} \mathrm{C}$ in a flowing nitrogen atmosphere of $60 \mathrm{ml} / \mathrm{min}$. The results for each of the twelve samples received January 15, 2013 are shown in Table 2-3. The initial mass loss is associated with the free water from the mix. The second mass loss beginning at approximately $400{ }^{\circ} \mathrm{C}$ is due to the thermal decomposition of the colemanite. Table 2-3 summarizes the mass change and hydrogen partial density for all twelve samples. The mass loss graphs for all twelve samples are in Appendix A.

In order to calculate the hydrogen partial density, it is assumed the average total mass loss for each sample in Table 2-3 is due to water, both free and from the decomposition of colemanite. The hydrogen partial density is calculated from the average mass loss (Table 2-3) and density of the sample (Table 2-2) as shown in equations $8-12$. Sample G3-M11-2000-B is used as the example calculation.

$$
\begin{aligned}
& \text { Moles } \mathrm{H}_{2} \mathrm{O} \text { in } 100 \mathrm{~g} \text { concrete }=\frac{\text { mass fraction } \mathrm{H}_{2} \mathrm{O}}{M \mathrm{M} \mathrm{H}_{2} \mathrm{O}}=\frac{31.41 \frac{\mathrm{g} \mathrm{H} \mathrm{H}_{2} \mathrm{O}}{\mathrm{gconcrete}}}{18.015 \mathrm{~g} \mathrm{~mol} \mathrm{H}_{2} \mathrm{O}}=1.74 \mathrm{molH}_{2} \mathrm{O}, \\
& \frac{\mathrm{g} \mathrm{H}}{\text { Mole } \mathrm{H}_{2} \mathrm{O}}=2.016 \mathrm{~g} \mathrm{H}, \\
& \mathrm{g} \mathrm{H} \text { in } 100 \mathrm{~g} \text { concrete }=1.74 \mathrm{~mol} \mathrm{H}_{2} \mathrm{O} \times 2.016 \mathrm{~g} \mathrm{H}=3.52 \mathrm{~g} \mathrm{H}, \\
& \text { Volume concrete }=\frac{\text { mass concrete }}{\text { density concrete }}=\frac{100 \mathrm{~g} \text { concrete }}{2.03 \frac{\mathrm{g}}{\mathrm{cm}^{3}}}=49.35 \mathrm{~cm}^{3} \text { concrete, } \\
& \mathrm{H} \text { density } \frac{\mathrm{g}}{\mathrm{ml}}=\frac{\text { mass } \mathrm{H}}{\text { volume concrete }}=\frac{3.52 \mathrm{~g} \mathrm{H}}{49.35 \mathrm{ml} \mathrm{concrete}}=7.12 \mathrm{E}-02 \frac{\mathrm{g} \mathrm{H}}{\mathrm{ml} \mathrm{Concrete} .} .
\end{aligned}
$$


Table 2-3. Mass loss from $25-650{ }^{\circ} \mathrm{C}$ for samples received on January 15, 2013

\begin{tabular}{|c|c|c|c|c|c|}
\hline Sample ID & $\begin{array}{c}\text { Mass Loss } \\
(\%) \\
25-175^{\circ} \mathrm{C}\end{array}$ & $\begin{array}{c}\text { Mass Loss } \\
(\%) \\
300-600{ }^{\circ} \mathrm{C}\end{array}$ & $\begin{array}{c}\text { Total Mass } \\
\text { Loss (\%) }\end{array}$ & $\begin{array}{l}\text { Average } \\
\text { Total Mass } \\
\text { Loss (\%) }\end{array}$ & $\begin{array}{c}\text { Total Hydrogen } \\
\text { Partial Density } \\
\left(\mathrm{g} / \mathrm{cm}^{3}\right)\end{array}$ \\
\hline G3-M11-2000-B-1 & 15.43 & 11.90 & 28.38 & \multirow{3}{*}{31.41} & \multirow{3}{*}{ 7.12E-02 } \\
\hline G3-M11-2000-B-2 & 18.95 & 11.32 & 32.34 & & \\
\hline G3-M11-2000-B-3 & 21.62 & 9.33 & 33.52 & & \\
\hline G3-M11-2000-M-1 & 26.08 & 9.42 & 37.42 & \multirow{3}{*}{31.69} & \multirow{3}{*}{ 7.25E-02 } \\
\hline G3-M11-2000-M-2 & 11.15 & 14.80 & 26.78 & & \\
\hline G3-M11-2000-M-3 & 17.62 & 11.30 & 30.86 & & \\
\hline G3-M11-2000-H-1 & 19.64 & 8.67 & 29.97 & \multirow{3}{*}{34.13} & \multirow{3}{*}{ 7.73E-02 } \\
\hline G3-M11-2000-H-2 & 24.79 & 8.17 & 34.95 & & \\
\hline G3-M11-2000-H-3 & 25.67 & 9.52 & 37.48 & & \\
\hline G3-M11-3000-B-1 & 9.50 & 17.66 & 28.00 & \multirow{3}{*}{29.72} & \multirow{3}{*}{ 6.65E-02 } \\
\hline G3-M11-3000-B-2 & 19.07 & 10.68 & 30.23 & & \\
\hline G3-M11-3000-B-3 & 18.64 & 10.14 & 30.92 & & \\
\hline G3-M11-3000-M-1 & 18.05 & 11.40 & 31.03 & \multirow{3}{*}{32.36} & \multirow{3}{*}{ 7.33E-02 } \\
\hline G3-M11-3000-M-2 & 18.80 & 11.89 & 32.64 & & \\
\hline G3-M11-3000-M-3 & 22.00 & 9.17 & 33.40 & & \\
\hline G3-M11-3000-H-1 & 14.88 & 14.62 & 30.69 & \multirow{3}{*}{31.53} & \multirow{3}{*}{ 7.26E-02 } \\
\hline G3-M11-3000-H-2 & 19.76 & 9.88 & 32.92 & & \\
\hline G3-M11-3000-H-3 & 19.15 & 9.53 & 30.97 & & \\
\hline G4-M1-2000-B-1 & 13.42 & 10.90 & 25.75 & \multirow{3}{*}{29.98} & \multirow{3}{*}{ 6.81E-02 } \\
\hline G4-M1-2000-B-2 & 16.09 & 19.19 & 34.21 & & \\
\hline G4-M1-2000-B-3 & 16.15 & 11.77 & 29.98 & & \\
\hline G4-M1-2000-M-1 & 18.37 & 10.55 & 31.03 & \multirow{3}{*}{30.14} & \multirow{3}{*}{ 6.85E-02 } \\
\hline G4-M1-2000-M-2 & 14.15 & 14.09 & 29.71 & & \\
\hline G4-M1-2000-M-3 & 15.17 & 13.08 & 29.69 & & \\
\hline G4-M1-2000-H-1 & 17.96 & 10.47 & 30.39 & \multirow{3}{*}{30.05} & \multirow{3}{*}{ 6.88E-02 } \\
\hline G4-M1-2000-H-2 & 15.82 & 11.26 & 29.13 & & \\
\hline G4-M1-2000-H-3 & 17.15 & 11.53 & 30.63 & & \\
\hline G5-M1-3000-B-1 & 17.74 & 10.97 & 30.24 & \multirow{3}{*}{32.88} & \multirow{3}{*}{ 7.47E-02 } \\
\hline G5-M1-3000-B-2 & 21.97 & 9.20 & 33.50 & & \\
\hline G5-M1-3000-B-3 & 22.72 & 9.94 & 34.89 & & \\
\hline G5-M1-3000-M-1 & 21.11 & 9.92 & 32.92 & \multirow{3}{*}{32.68} & \multirow{3}{*}{ 7.42E-02 } \\
\hline G5-M1-3000-M-2 & 16.55 & 12.58 & 30.82 & & \\
\hline G5-M1-3000-M-3 & 21.68 & 10.53 & 34.29 & & \\
\hline G5-M1-3000-H-1 & 21.52 & 8.54 & 32.19 & \multirow{3}{*}{31.92} & \multirow{3}{*}{ 7.24E-02 } \\
\hline G5-M1-3000-H-2 & 22.15 & 9.32 & 33.17 & & \\
\hline G5-M1-3000-H-3 & 18.42 & 10.11 & 30.39 & & \\
\hline
\end{tabular}




\subsection{Boron Partial Density}

Subsamples of the samples received on January 15, 2013 were crushed, dried in an oven to remove moisture, and digested in triplicate using the ASTM method for trace metals analysis in limestone. ${ }^{6}$ Aliquots of each sample were weighed in separate beakers and then $10 \mathrm{ml}$ of $\mathrm{HCl}$ and $4 \mathrm{ml}$ of $\mathrm{HNO}_{3}$ were added. The acid mixture was heated at $85{ }^{\circ} \mathrm{C}$ for 60 minutes on a hotplate, with the sample covered with a watch glass. After heating was complete, the sample cooled for an additional 60 minutes to ensure complete boron dissolution. The sample was then diluted up to a final volume of $100 \mathrm{ml}$ with deionized water. The samples were analyzed on the Agilent 730 Inductively Coupled Plasma-Optical Emission Spectrometer (ICP-OES). Boron was calibrated using a High Purity NIST traceable standard (Lot 1204016), Appendix B: Boron Certificate of Analysis. An internal standard (Yttrium) was used to compensate for matrix effects. The dissolution method prescribed in the ASTM method resulted in complete dissolution of the samples. Table 2-4 is the analytical results of the dissolution of colemanite concrete using the prescribed ASTM method.

Table 2-4. Boron results for colemanite concrete samples received January 15, 2013

\begin{tabular}{|c|c|c|}
\hline Sample ID & $\begin{array}{l}\text { Average Boron } \\
\text { Content [wt \%] }\end{array}$ & $\begin{array}{c}\text { Average Partial Boron } \\
\text { Density }\left[\mathrm{g} / \mathrm{cm}^{3}\right]\end{array}$ \\
\hline G3-M11-2000-B & 8.40 & $1.70 \mathrm{E}-01$ \\
\hline G3-M11-2000-M & 8.74 & 1.79E-01 \\
\hline G3-M11-2000-H & 7.96 & $1.61 \mathrm{E}-01$ \\
\hline G3-M11-3000-B & 8.46 & 1.69E-01 \\
\hline G3-M11-3000-M & 8.10 & 1.64E-01 \\
\hline G3-M11-3000-H & 8.27 & $1.70 \mathrm{E}-01$ \\
\hline G4-M1-2000-B & 8.49 & $1.72 \mathrm{E}-01$ \\
\hline G4-M1-2000-M & 8.24 & $1.67 \mathrm{E}-01$ \\
\hline G4-M1-2000-H & 8.40 & $1.72 \mathrm{E}-01$ \\
\hline G5-M1-3000-B & 8.59 & $1.74 \mathrm{E}-01$ \\
\hline G5-M1-3000-M & 8.16 & $1.66 \mathrm{E}-01$ \\
\hline G5-M1-3000-H & 7.86 & 1.59E-01 \\
\hline
\end{tabular}

The average partial boron density for each sample is calculated using the calculated colemanite concrete density for each sample in Table 2-2. An example calculation for the partial density of sample G3-M11-2000-B is shown in equations $13-15$ :

Mass boron in $100 \mathrm{~g}$ concrete from Table 2-4 = $8.40 \mathrm{~g}$

Volume concrete $=\frac{\text { mass concrete }}{\text { density concrete }}=\frac{100 \mathrm{~g} \text { concrete }}{2.03 \frac{\mathrm{g}}{\mathrm{cm}^{3}}}=49.35 \mathrm{~cm}^{3}$ concrete

Boron density $\frac{\mathrm{g}}{\mathrm{ml}}=\frac{\text { mass } B}{\text { volume concrete }}=\frac{8.40 \mathrm{~g} \mathrm{~B}}{49.35 \mathrm{~cm}^{3}}=1.70 \mathrm{E}-01 \frac{\mathrm{g} \text { boron }}{\mathrm{cm}^{3} \text { Concrete }}$

Comparing the results in Table 2-4 to the acceptability limits in Table 1-1, samples G3-M112000-H, G3-M11-3000-M, and G5-M1-3000-H are below the limit $\left(1.65 \mathrm{E}-01 \mathrm{~g} / \mathrm{cm}^{3}\right)$ for the average partial boron density. 


\subsection{Conclusions}

The lower limits and measured values for the total density, hydrogen partial density, and boron partial density are presented in Table 3-1. For all the samples tested, the total density and the hydrogen partial density met or exceeded the lower bounds specified in Reference 1 . All of the samples met or exceeded the boron partial density lower bound with the exception of samples G3-M11-2000-H, G3-M11-3000-M, and G5-M1-3000-H which are below the limit of 1.65E-01 $\mathrm{g} / \mathrm{cm}^{3}$.

Table 3-1. Lower Bounds and Average Measured Values for the Total Density, Hydrogen Partial Density, and Boron Partial Density for the Colemanite Concrete Samples Received January 15, 2013

\begin{tabular}{|c|c|c|c|c|c|c|}
\hline \multirow[t]{2}{*}{ Sample ID } & \multicolumn{2}{|c|}{$\begin{array}{c}\text { Total Density Lower } \\
\text { Bound } \\
{\left[\mathrm{g} / \mathrm{cm}^{3}\right]} \\
\end{array}$} & \multicolumn{2}{|c|}{$\begin{array}{c}\text { Hydrogen Partial } \\
\text { Density Lower Bound } \\
{\left[\mathrm{g} / \mathrm{cm}^{3}\right]}\end{array}$} & \multicolumn{2}{|c|}{$\begin{array}{c}\text { Boron Partial Density } \\
\text { Lower Bound } \\
{\left[\mathrm{g} / \mathrm{cm}^{3}\right]}\end{array}$} \\
\hline & Limit & Measured & Limit & Measured & Limit & Measured \\
\hline G3-M11-2000-B & \multirow{12}{*}{1.88} & 2.03 & \multirow{12}{*}{ 6.04E-02 } & 7.12E-02 & \multirow{12}{*}{$1.65 \mathrm{E}-01$} & 1.70E-01 \\
\hline G3-M11-2000-M & & 2.04 & & 7.25E-02 & & 1.79E-01 \\
\hline G3-M11-2000-H & & 2.02 & & 7.73E-02 & & $1.61 \mathrm{E}-01$ \\
\hline G3-M11-3000-B & & 2.00 & & $6.65 \mathrm{E}-02$ & & $1.69 \mathrm{E}-01$ \\
\hline G3-M11-3000-M & & 2.02 & & 7.33E-02 & & 1.64E-01 \\
\hline G3-M11-3000-H & & 2.06 & & $7.26 \mathrm{E}-02$ & & 1.70E-01 \\
\hline G4-M1-2000-B & & 2.03 & & 6.81E-02 & & 1.72E-01 \\
\hline G4-M1-2000-M & & 2.03 & & 6.85E-02 & & 1.67E-01 \\
\hline G4-M1-2000-H & & 2.05 & & $6.88 \mathrm{E}-02$ & & $1.72 \mathrm{E}-01$ \\
\hline G5-M1-3000-B & & 2.03 & & $7.47 \mathrm{E}-02$ & & $1.74 \mathrm{E}-01$ \\
\hline G5-M1-3000-M & & 2.03 & & 7.42E-02 & & $1.66 \mathrm{E}-01$ \\
\hline G5-M1-3000-H & & 2.03 & & 7.24E-02 & & 1.59E-01 \\
\hline
\end{tabular}




\subsection{References}

1. Wead, R., "Radiation Shielding and Fixed Neutron Absorber Panel Material and Inspection Requirements," DCS01-ZMJ-DS-SPE-M-19109-2, Revision 2, 2007.

2. $\quad$ "Equipment Calibration Services / Material Testing," WTA-040-8b, Section 9 (q), April 13, 2012.

3. "Standard Test Method for Density, Absorption, and Voids in Hardened Concrete," ASTM International, ASTM C 642-06.

4. "Standard Test Method for Compositional Analysis by Thermogravimetry," ASTM International, ASTM E 1131-08.

5. Waclawska, I., Stoch, L., Paulik, J., and Pauliik, F., "Thermal Decomposition of Colemanite," Thermochimica Acta, 126, 307-18 (1988).

6. "Major and Trace Elements in Limestone and Lime by Inductively Coupled PlasmaAtomic Emission Spectroscopy (ICP) and Atomic Absorption (AA)," ASTM International, ASTM C 1301-95 (reapproved 2009). 
SRNL-STI-2013-00078

Revision 0

Appendix A. TGA Graphs for Samples Received January 15, 2013 


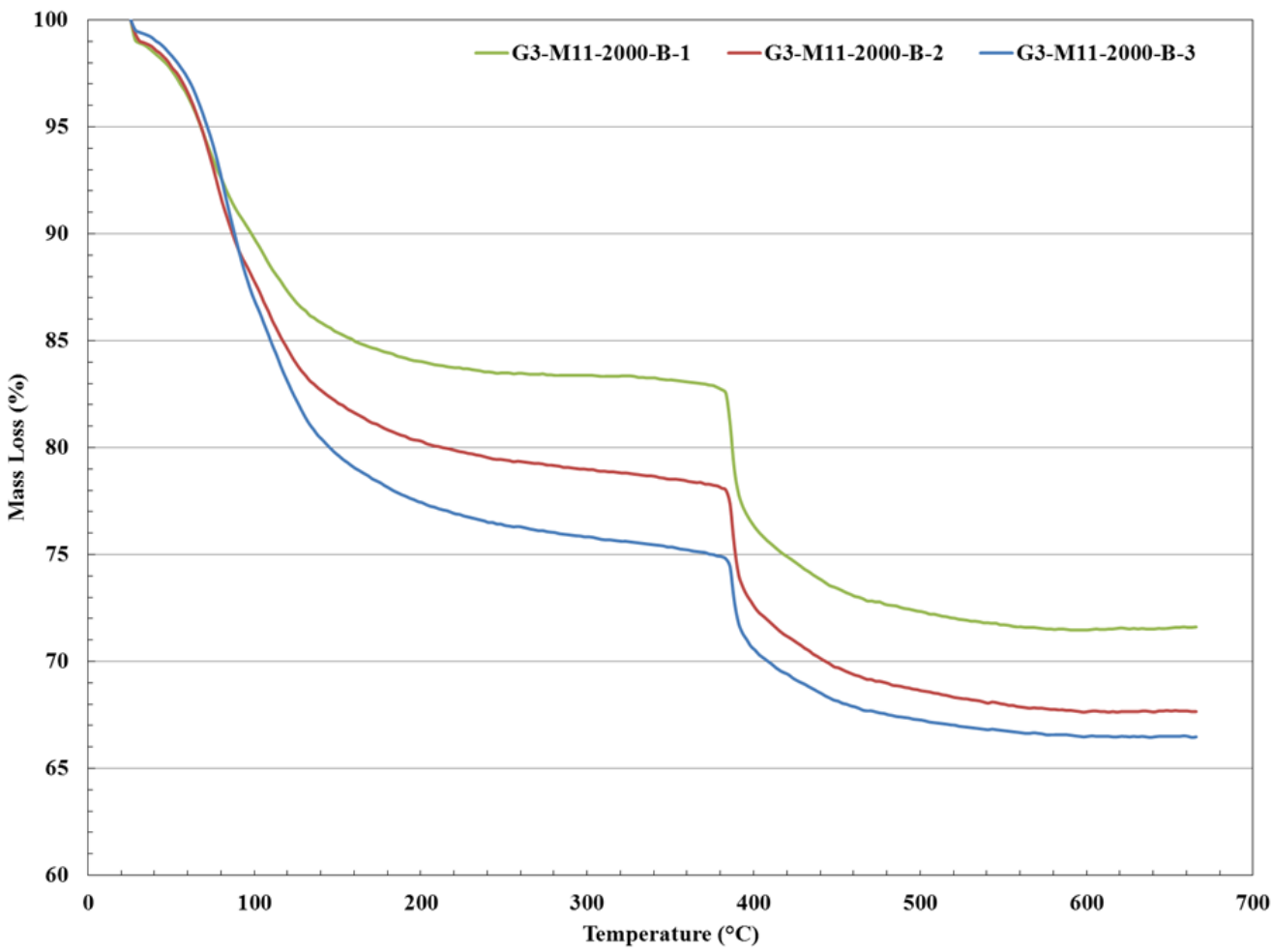

Figure A-1. TGA curve showing the mass loss for sample G3-M11-2000-B

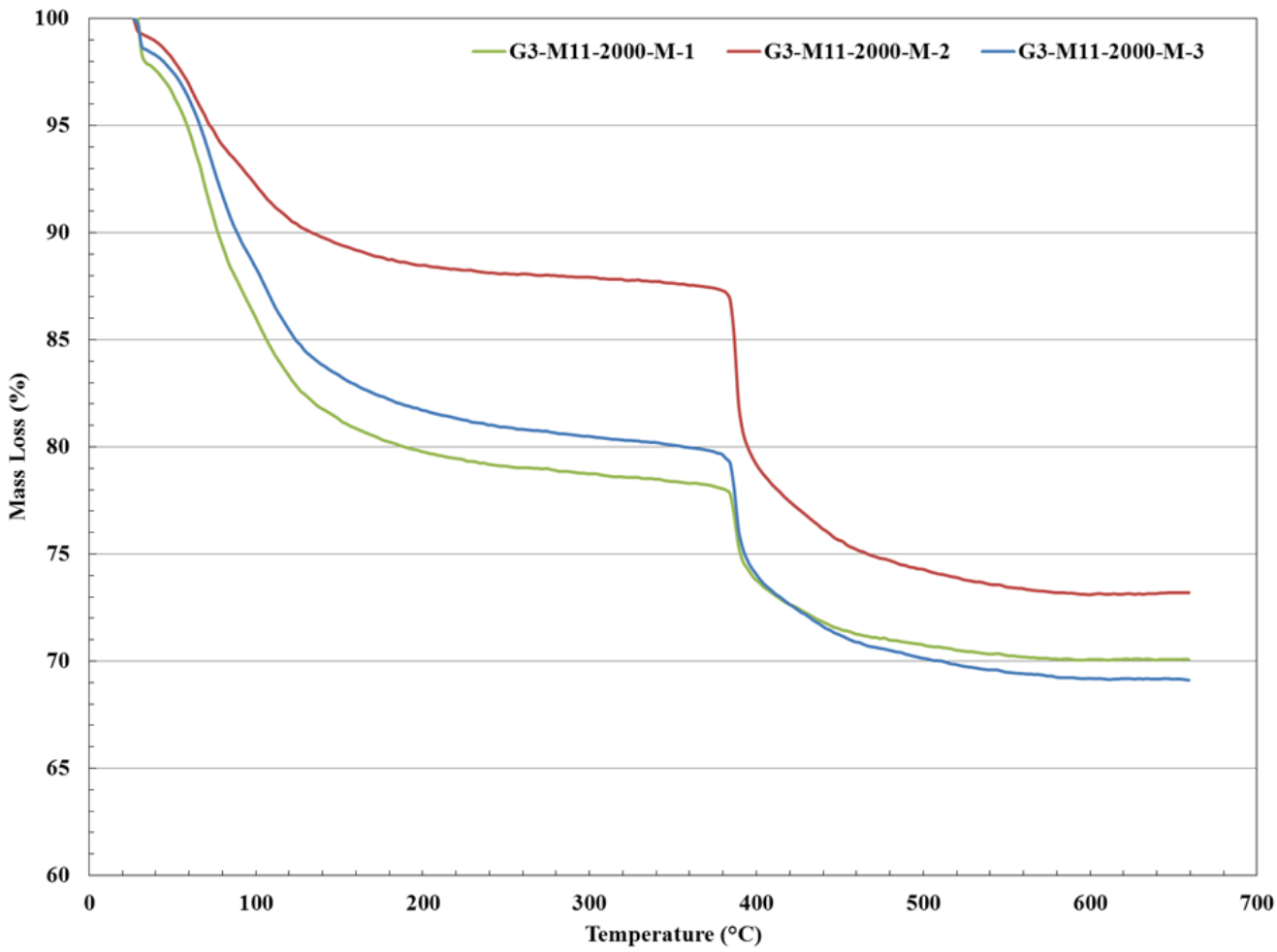

Figure A-2. TGA curve showing the mass loss for sample G3-M11-2000-M 


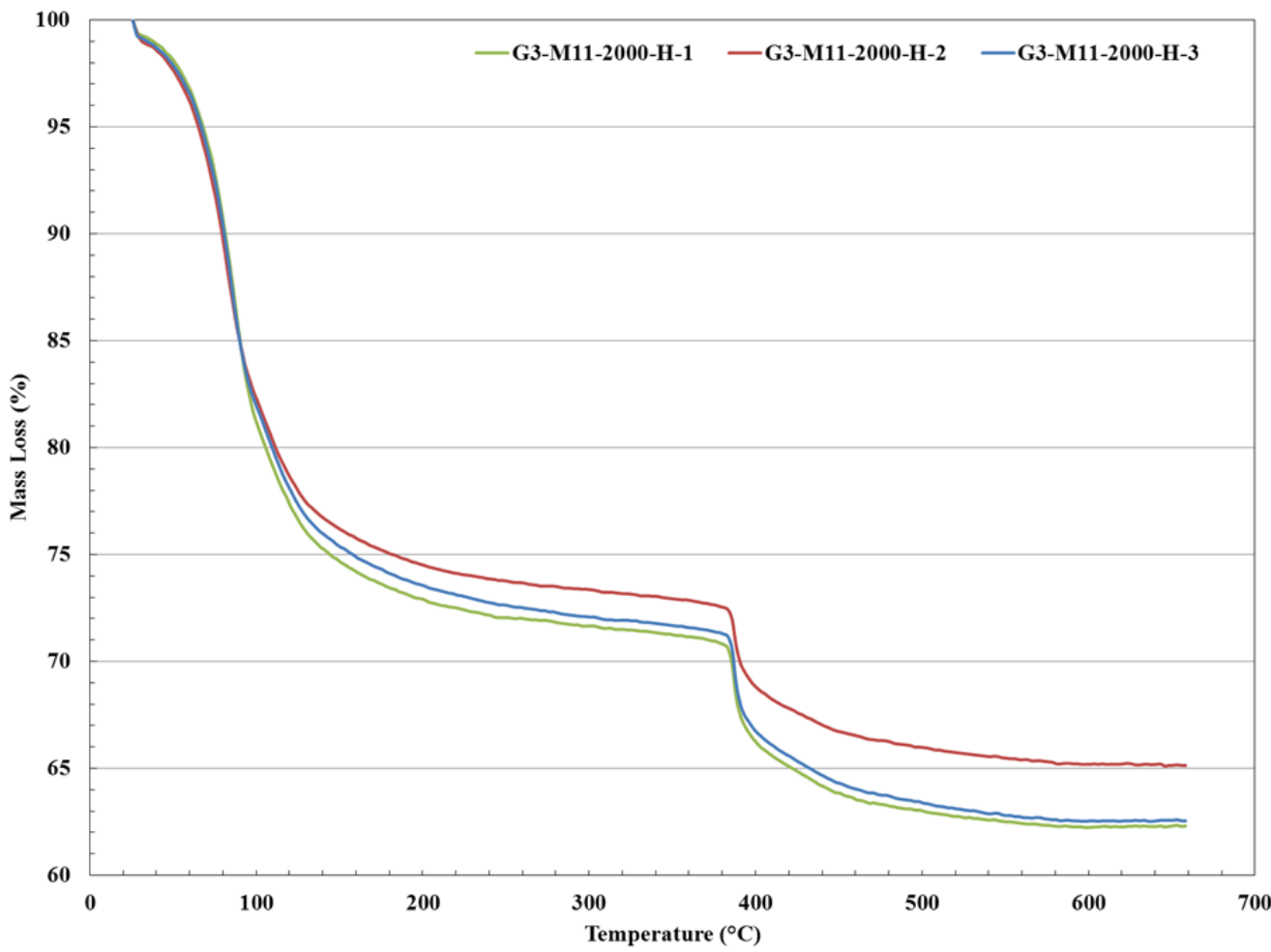

Figure A-3. TGA curve showing the mass loss for sample G3-M11-2000-H

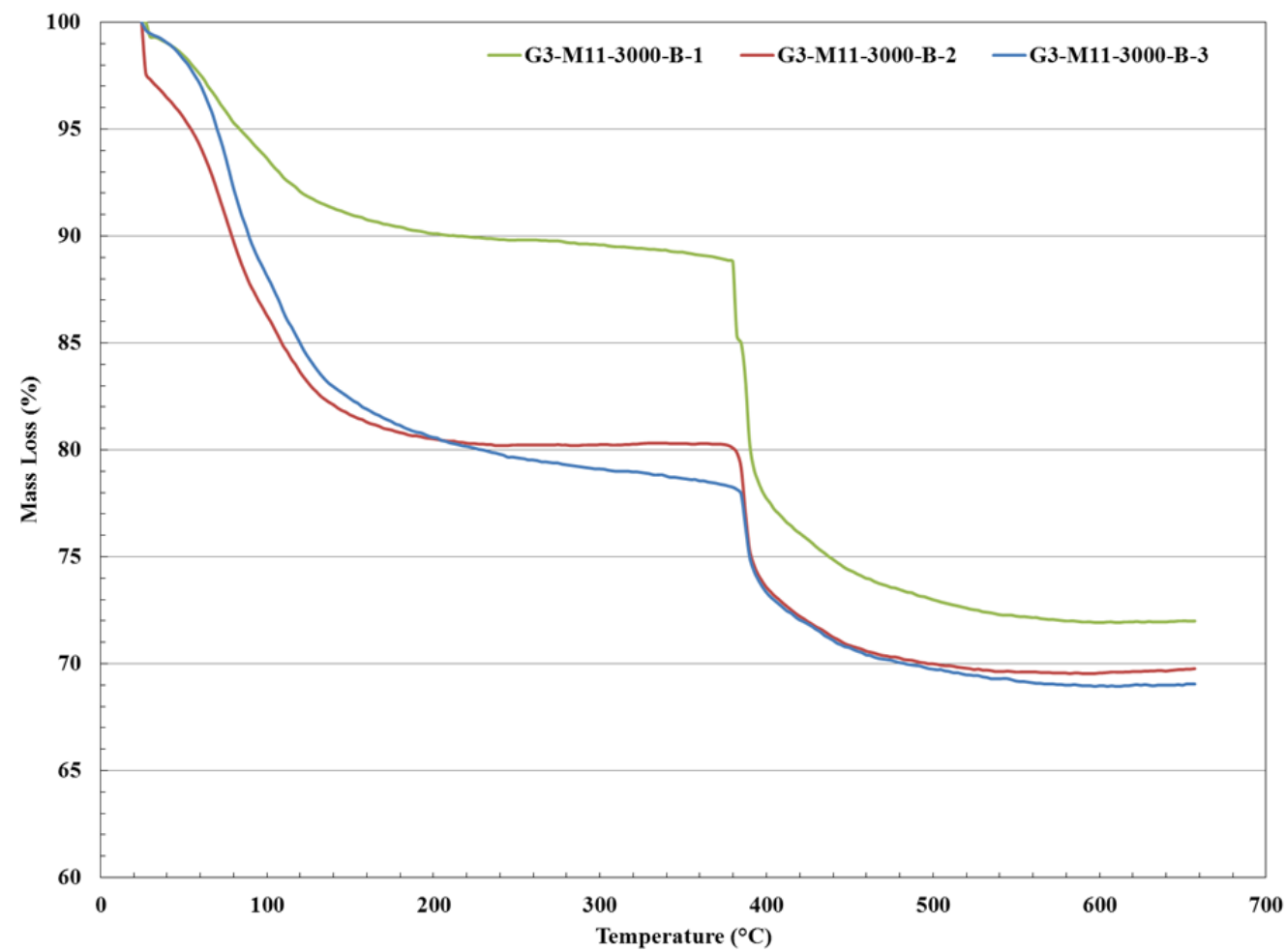

Figure A-4. TGA curve showing the mass loss for sample G3-M11-3000-B 


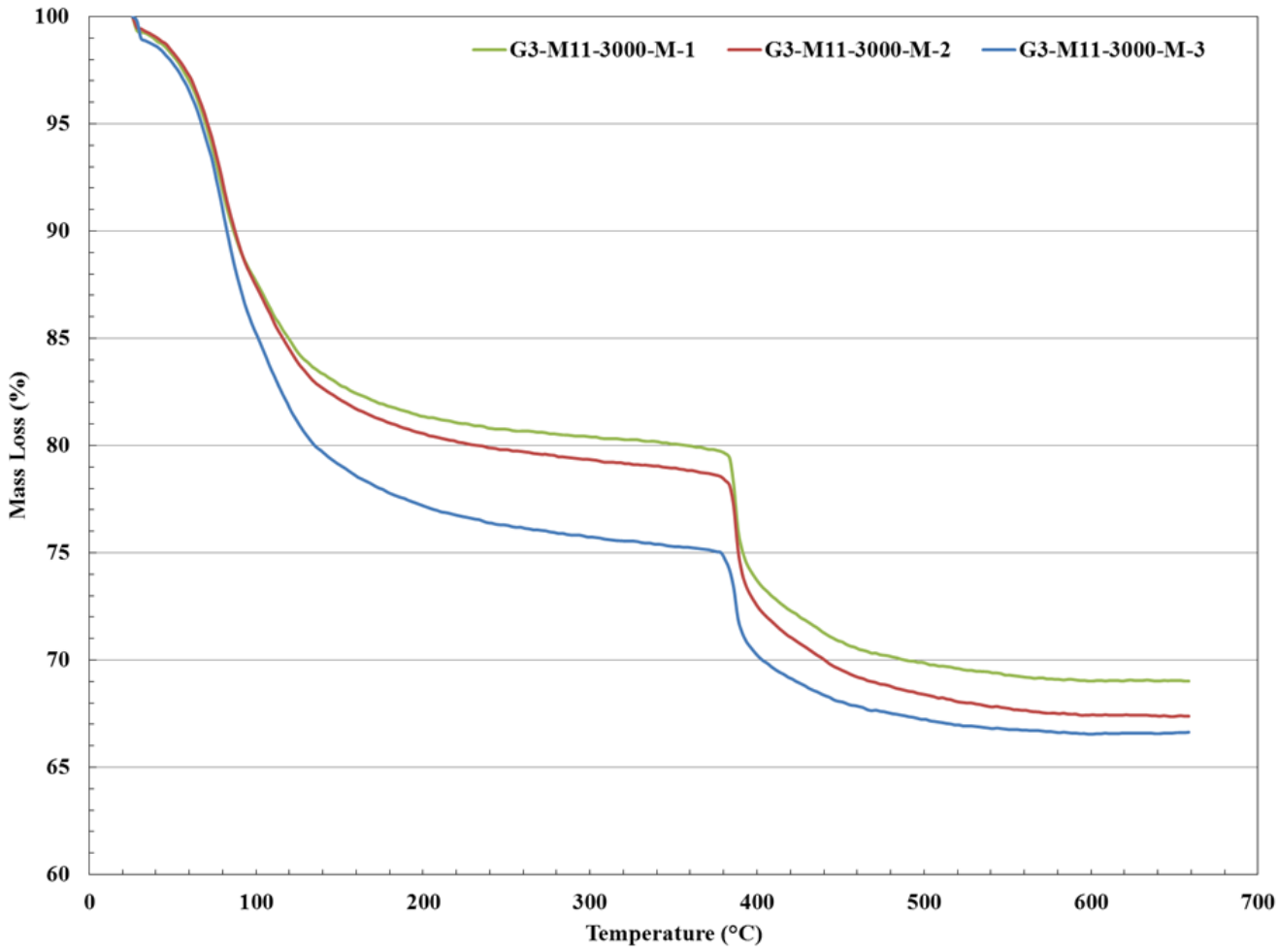

Figure A-5. TGA curve showing the mass loss for sample G3-M11-3000-M

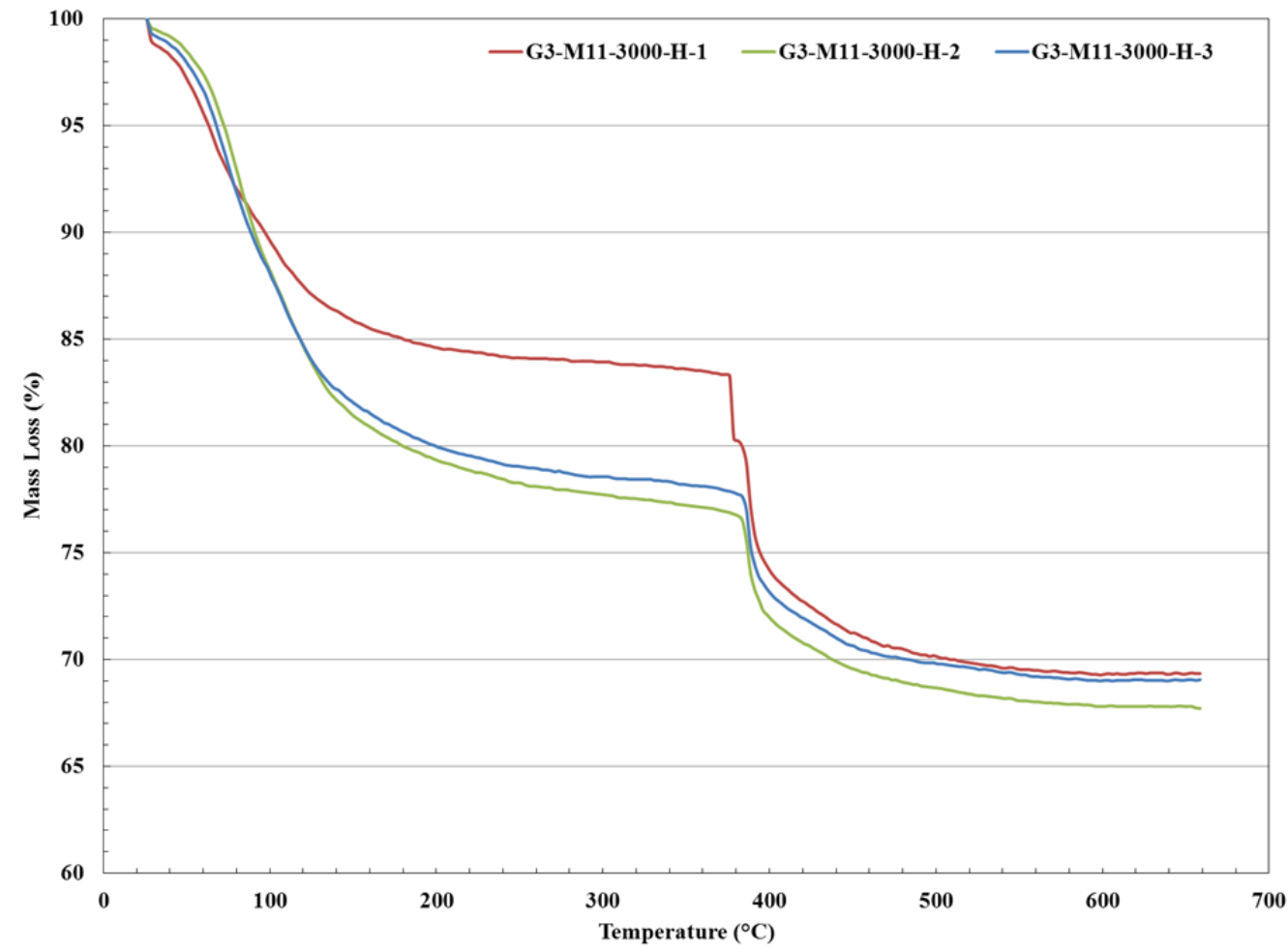

Figure A-6. TGA curve showing the mass loss for sample G3-M11-3000-H 


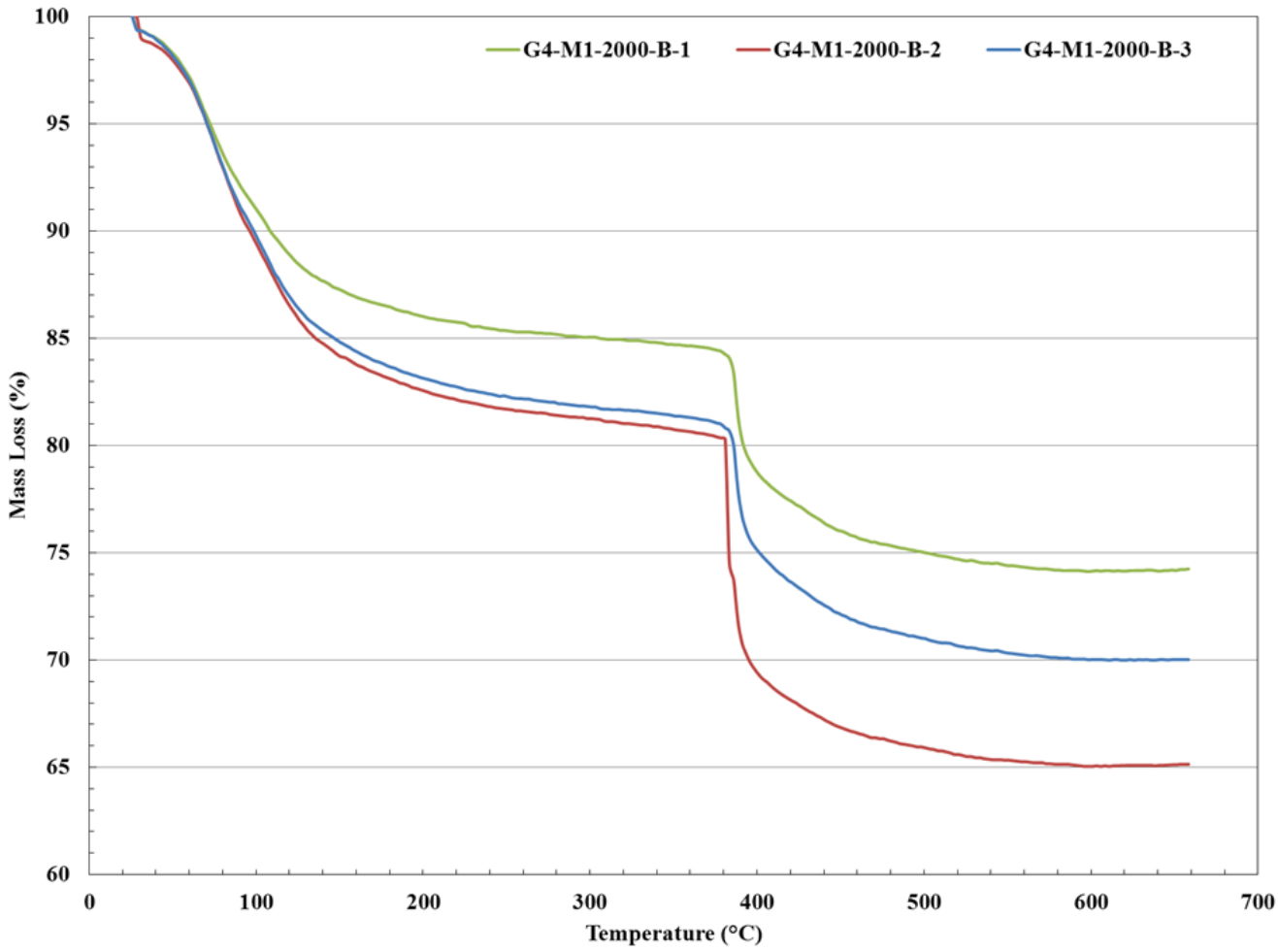

Figure A-7. TGA curve showing the mass loss for sample G4-M1-2000-B

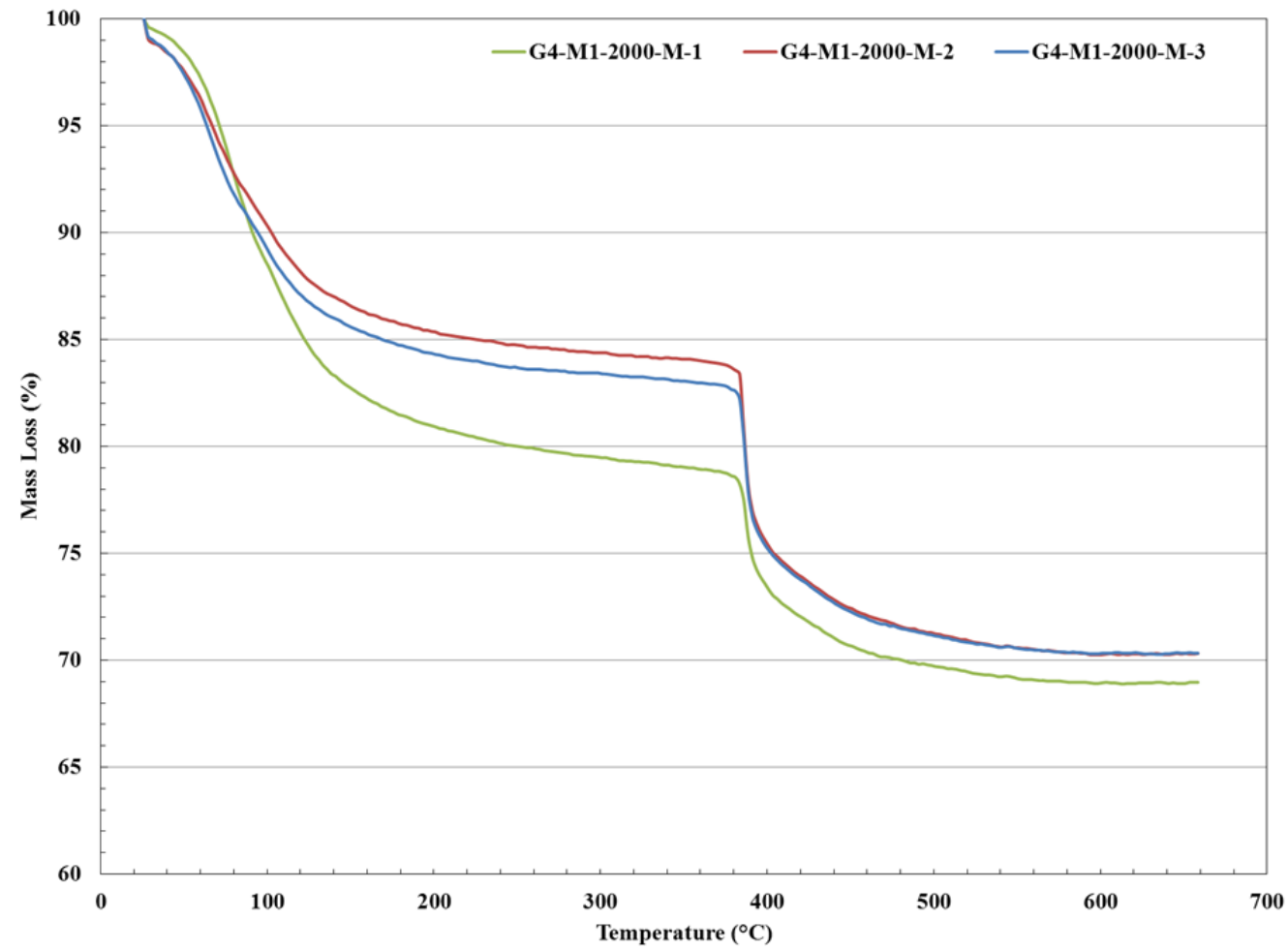

Figure A-8. TGA curve showing the mass loss for sample G4-M1-2000-M 


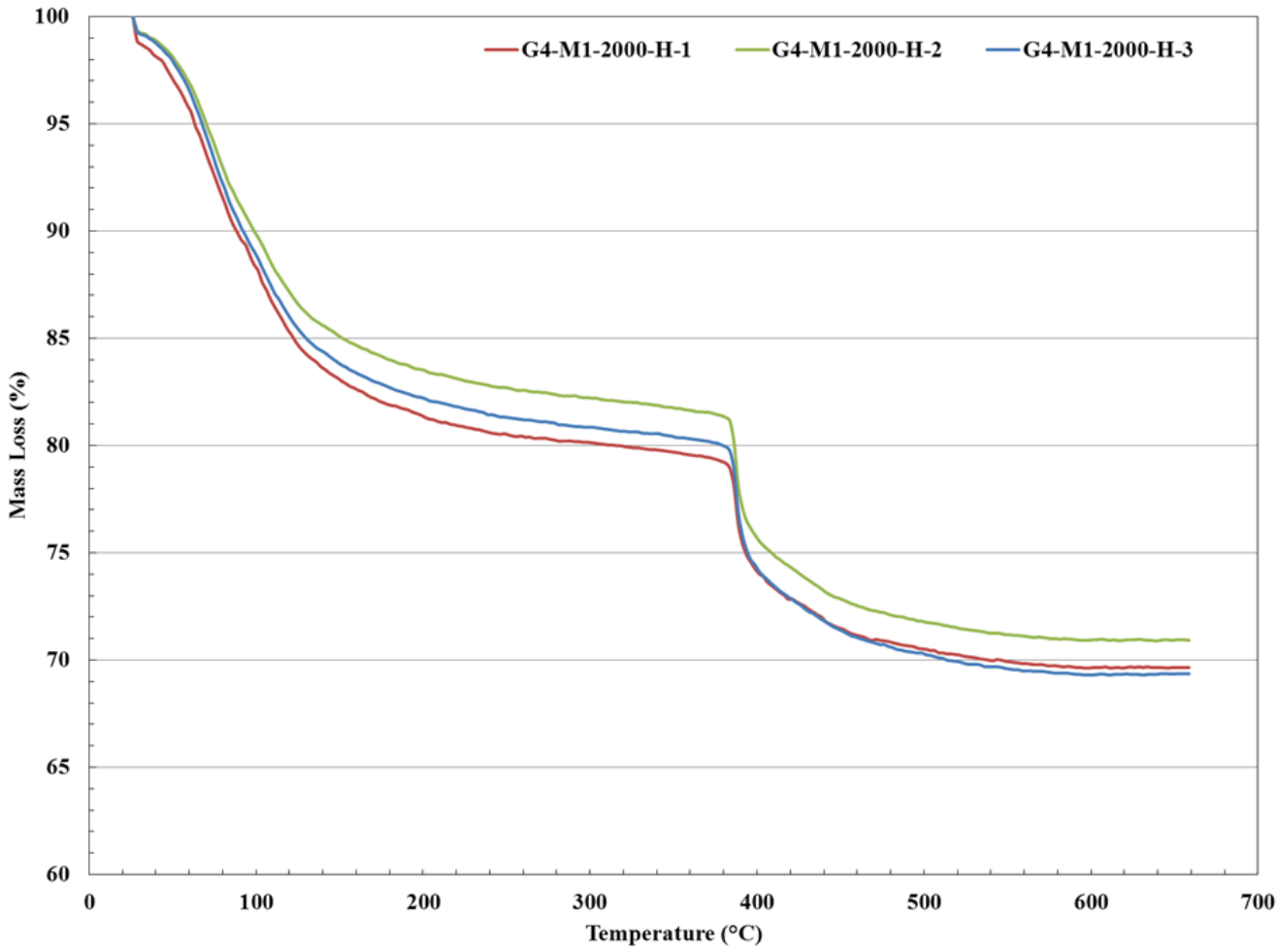

Figure A-9. TGA curve showing the mass loss for sample G4-M1-2000-H

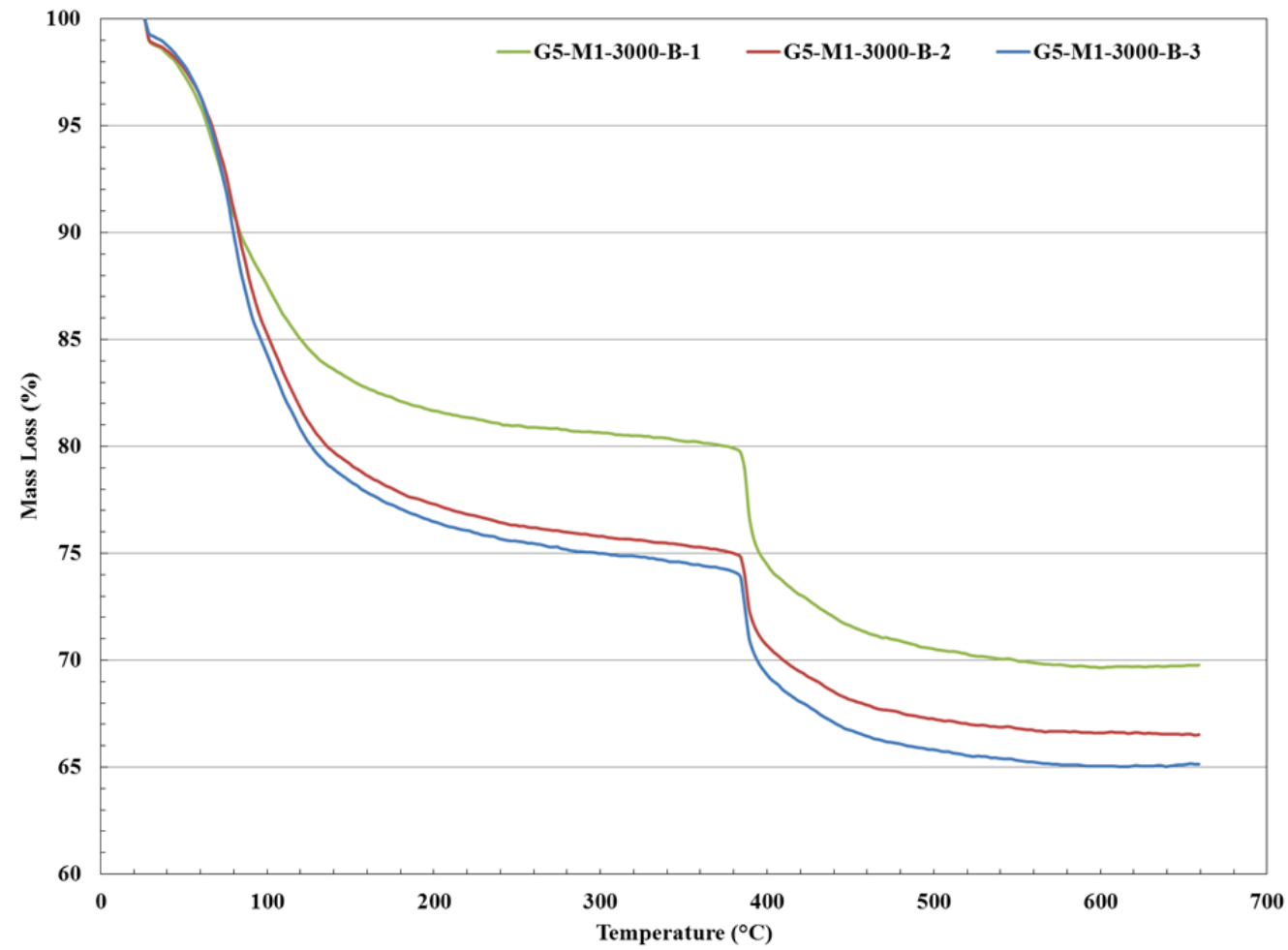

Figure A-10. TGA curve showing the mass loss for sample G5-M1-3000-B 


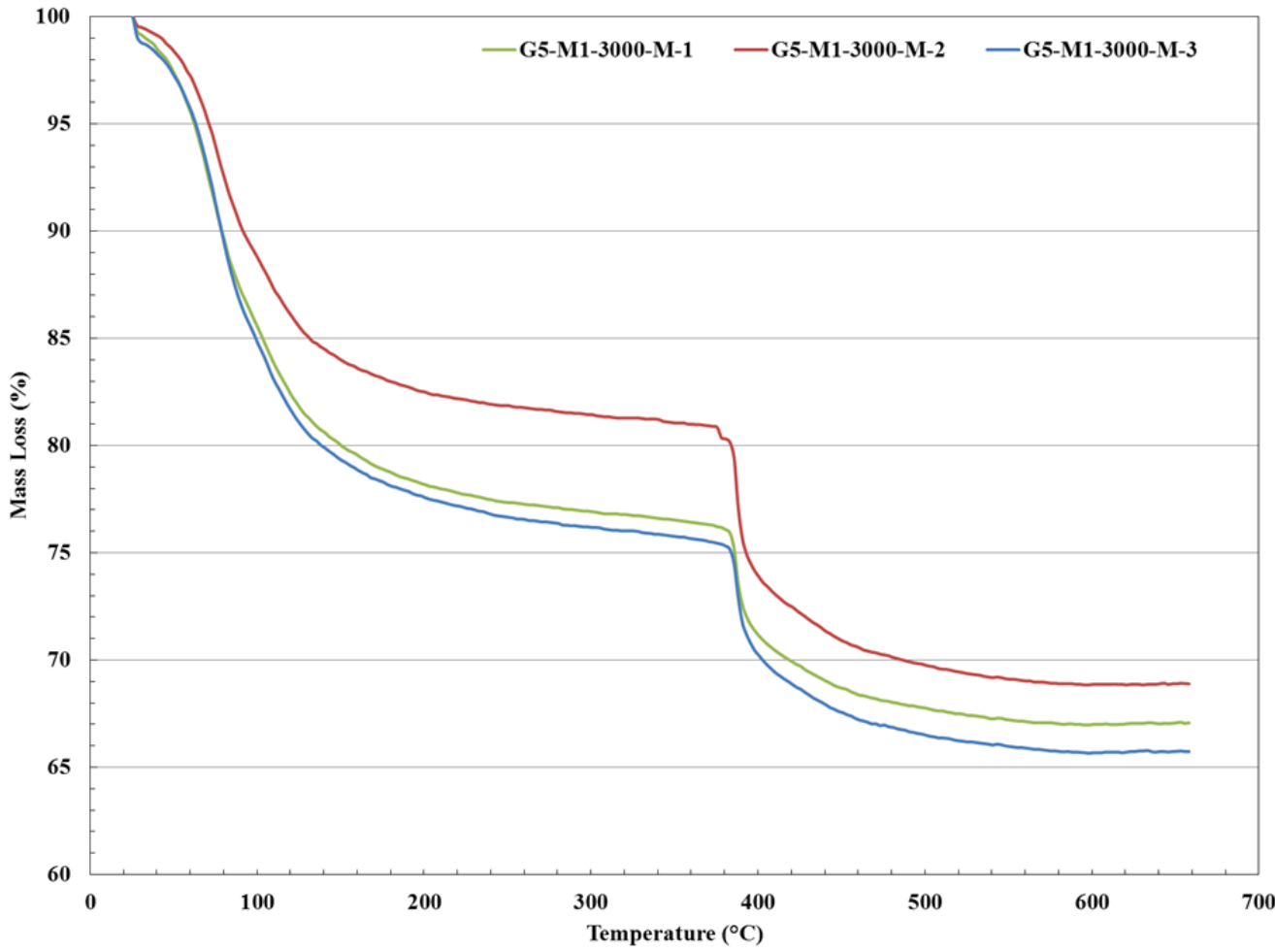

Figure A-11. TGA curve showing the mass loss for sample G5-M1-3000-M

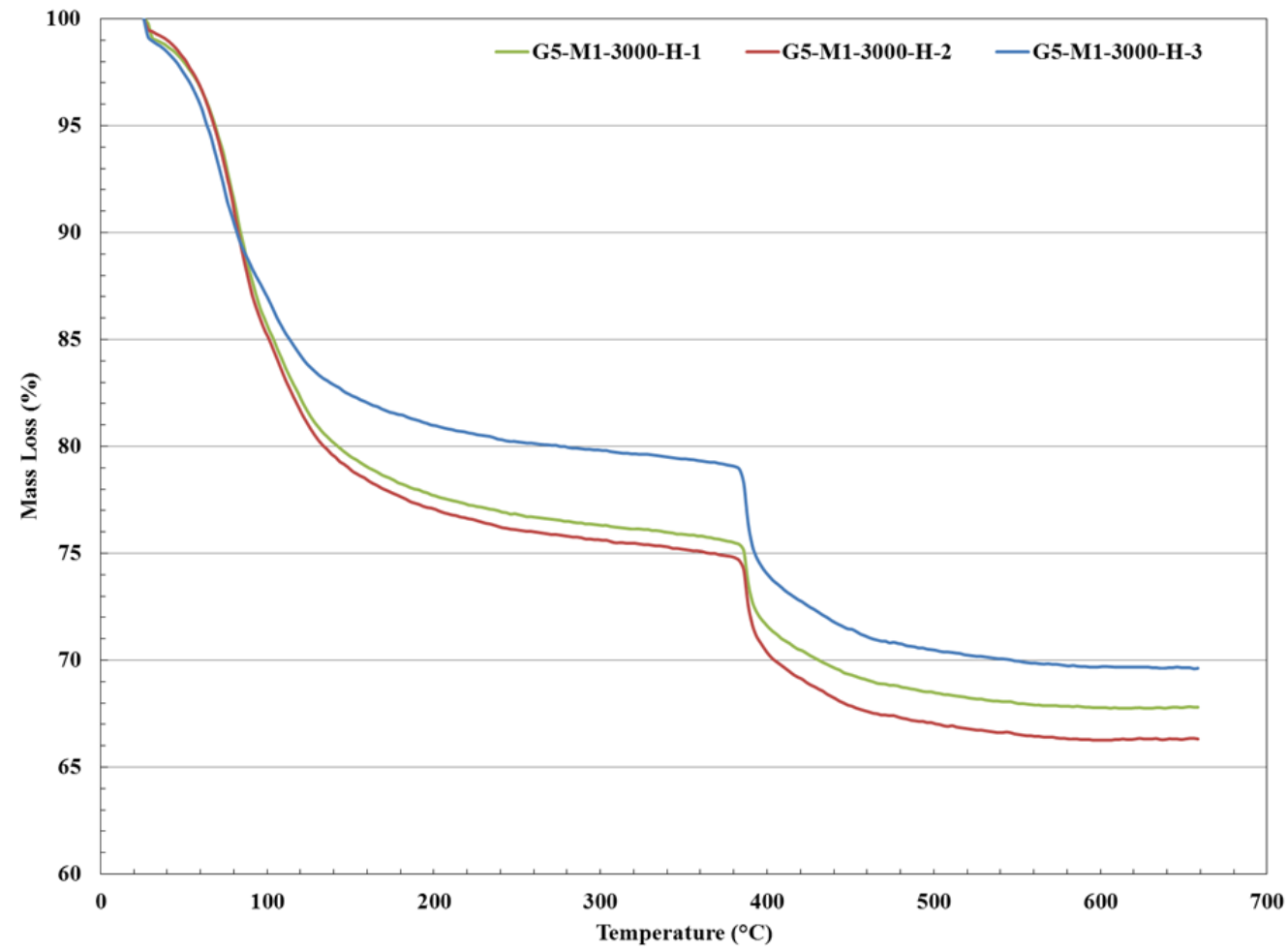

Figure A-12. TGA curve showing the mass loss for sample G5-M1-3000-H 
SRNL-STI-2013-00078

Revision 0

Appendix B. Boron Certificate of Analysis 


\title{
I/ \\ wmehighparcyatandards com
}

\section{Certifícate of Analpsis}

\section{Product Description:}

$\begin{array}{llll}\text { Name: } & \text { Boron } & \text { Source Material: } & \text { Boric Acid } \\ \text { Part Number: } & 10007-4 & \text { Material Purity: } & 99.999 \% \\ \text { Lot Number: } & 1204016 & \text { Matrix: } & \mathrm{H}_{2} \mathrm{O}\end{array}$

\section{Certified Value:}

$1000 \mu \mathrm{g} / \mathrm{mL} \pm 3 \mu \mathrm{g} / \mathrm{mL}$

The Certified value is based on gravimetric and volumetric preparation, and confirmed against SRM 3107 (lot number 070514 ) by inductively coupled plasma optical emission spectrometry (ICP-OES) using an internal laboratory-developed method. The uncertainty in the certified value is calculated for a $95 \%$ confidence interval and coverage factor $k$ is about 2 .

Density:

$$
1.000 \mathrm{~g} / \mathrm{mL} \pm 0.002 \mathrm{~g} / \mathrm{mL} @ 22.8^{\circ} \mathrm{C}
$$

\section{Uncertified Values:}

\section{Titration Value: $\quad 994.97 \mu \mathrm{g} / \mathrm{mL}$}

Trace Metal Impurity Scan: The data reported are based upon a scan of this specific lot at $1000 \mu \mathrm{g} / \mathrm{mL}$ via ICP analysis. The values are reported in $\mu \mathrm{g} / \mathrm{L}$.

\begin{tabular}{|c|c|c|c|c|c|c|c|c|c|c|c|c|c|}
\hline $\mathrm{Ag}$ & 0.02 & $\mathrm{Cu}$ & $<$ & 0.1 & $\mathbf{L i}$ & $<$ & 1 & $\mathbf{R b}$ & $<$ & $0.0 ?$ & Th & $<$ & 0.02 \\
\hline $\mathrm{Al}$ & 0.1 & Dy & $<$ & 0.02 & $\mathrm{Lu}$ & $<$ & 0.02 & Re & $<$ & 0.02 & $\mathrm{~T}$ & $<$ & 0.02 \\
\hline As & 0.05 & Er & $<$ & 0.02 & $\mathrm{Mg}$ & $<$ & 0.5 & $\mathbf{R h}$ & $<$ & 0.02 & $\pi$ & $<$ & 0.02 \\
\hline Au & 0.02 & Eu & $<$ & 0.02 & $\mathrm{Mn}$ & $<$ & 0.5 & $\mathbf{R u}$ & $<$ & 0.02 & $\mathrm{Tm}$ & $<$ & 0.02 \\
\hline B & $\mathrm{M}$ & $\mathrm{Fe}$ & $<$ & 5 & Mo & $<$ & 0.02 & Sb & $<$ & 0.02 & $\mathbf{U}$ & $<$ & 0.1 \\
\hline Ba & 0.1 & $\mathrm{Ga}$ & $<$ & 0.02 & $\mathrm{Na}$ & $<$ & 10 & $\mathrm{Sc}$ & $<$ & 0.02 & v & $<$ & 0.05 \\
\hline $\mathrm{Be}$ & 0.02 & Gd & $<$ & 0.02 & $\mathrm{Nb}$ & $<$ & 0.02 & $\mathrm{Se}$ & $<$ & 0.1 & w & $<$ & 0.02 \\
\hline $\mathrm{Bi}$ & 0.02 & $\mathrm{Ge}$ & $<$ & 0.02 & Nd & $<$ & 0.02 & $\mathbf{S i}$ & $<$ & 5 & $\mathrm{Y}$ & $<$ & 0.02 \\
\hline $\mathrm{Ca}$ & 5 & $\mathrm{Hf}$ & $<$ & 0.02 & $\mathrm{Ni}$ & $<$ & 0.02 & Sm & $<$ & 0.02 & $\mathrm{Yb}$ & $<$ & 0.02 \\
\hline $\mathrm{Cd}$ & 0.02 & Ho & $<$ & 0.02 & Os & $<$ & 0.02 & $\mathrm{Sn}$ & $<$ & 1 & $\mathrm{Zn}$ & $<$ & 0.1 \\
\hline $\mathrm{Ce}$ & 0.02 & In & $<$ & 0.02 & $\mathrm{~Pb}$ & $<$ & 0.05 & $\mathrm{Sr}$ & $<$ & 0.02 & $\mathrm{Zr}$ & $<$ & 0.02 \\
\hline Co & 0.05 & Ir & $<$ & 0.02 & Pd & $<$ & 0.02 & $\mathrm{Ta}$ & $<$ & 0.02 & & & \\
\hline $\mathrm{Cr}$ & 0.1 & K & $<$ & 1 & $\mathrm{Pt}$ & $<$ & 0.02 & $\mathrm{~Tb}$ & $<$ & 0.02 & & & \\
\hline $\mathrm{Cs}$ & 0.02 & La & $<$ & 0.02 & $\mathrm{Pt}$ & $<$ & 0.02 & $\mathrm{Te}$ & $<$ & 0.02 & & & \\
\hline
\end{tabular}

Preparation Information:

The standard solution is prepared using high purity materials and assayed by analytical methods for conformity prior to use. This standard was prepared using the methods developed at NIST for SRM Spectrometric Standard Solutions under appropriate laboratory conditions.

The matrix is 18 megaohm deionized water.

Stability of this product is based upon rigorous short term and long term testing of the solution for the certified value. This testing includes, but is not limited to, the effect of temperature and packaging on the product.

Intended Use:

This Certified Reference Material (CRM) is intended for use as a calibration standard for the quantitative determination of boron, calibration of instruments such as ICPOES, ICPMS, AAS and XRF, and validation of analytical methods. It also can be used in EPA, ASTM and other methods.

\author{
Lot No.: 1204016 \\ Rev. No: 5.00 \\ Page 1 of 2
}


Traceability Information:

The traceability of this standard is maintained through an unbroken chain of comparisons to appropriate standards with suitable procedure and measurement uncertainties. The maintenance of the base and derived units of International System of Units (SI) with traceability of measurement results (contemporary metrology)

to SI ensures their comparability over time as follows.

a. Standard Weight and Analytical Balance

The standard weights (NBS weights Inventory No 20231A) are calibrated every two years by South Carolina Metrology Laboratory that is a participant in "NIST Weights and Measures Measurement Assurance Program" with a certificate of measurement traceability to NIST primary standards.

The balances are calibrated yearly by the ISO 17025 accredited metrology service, and are verified weekly by an in-house method using standard weights.

b. Volumetric Device

The calibration of volumetric vessels is checked annually using the NBS 602 method.

c. Thermometer

The standard thermometers are calibrated every year by the ISO 17025 accredited metrology service. The thermometers used in-house are verified against the standard thermometers yearly.

d. Calibration Standards:

The Calibration Standard is directly traceable to SRM 3100 Series Spectrometric Standard Solutions.

Packaging and Storage Conditions:

The standard is packaged in a pre-cleaned polyethylene bottle. To maintain the integrity of this product, the solution should be kept tightly capped and stored under normal laboratory conditions.

Refer to Material Safety Datasheet (MSDS) for hazardous information.

Expiration Information:

The expiry date is guaranteed to be valid for eighteen months from the shipping date provided. For this reason, standards from the same lot may have differeat expiration dates.

$\begin{array}{ll}\text { Preparation Date: } & \text { February 9,2012 } \\ \text { Shipped Date: } & \text { MAR } 092012 \\ \text { Expiration Date: } & \text { SEP } 092013\end{array}$

Expiration Date:

February 29, 2012

Quality Information:

(ACLSS)

ISO/IEC 17025:2005 Accreditation Certificate Number AT-1529
(aC1ass

ISO Guide 34:2009 (RMP) Accreditation Certificate Number AR-1436

\section{Coing 1 .}

Vanny T. Yib,

Inerganic Laboratory Manager

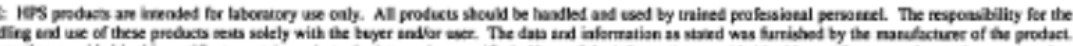

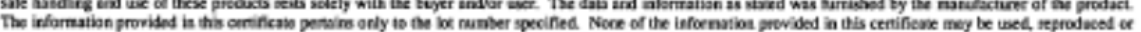

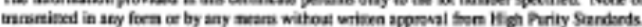

Lot No.: 1204016

Rev. No: 5.0 .0
Page 2 of 2 


\section{Distribution:}

D.R. Best, 999-W

T.B. Brown, 773-A

D.R. Click, 773-A

A.D. Cozzi, 999-W

S. D. Fink, 773-A

K.M. Fox, 999-W

C. C. Herman, 999-W

E.N. Hoffman, 999-W

S. L. Marra, 773-A

F. M. Pennebaker, 773-42A

M.M. Reigel, 999-W

J.A. Spear, 235-11F

J. P. Vaughan, 773-41A 